\title{
XXXII. The distribution of the active deposits of radium, thorium, and actinium in electric fields
}

\section{G.H. Briggs B.Sc.}

To cite this article: G.H. Briggs B.Sc. (1921) XXXII. The distribution of the active deposits of radium, thorium, and actinium in electric fields, Philosophical Magazine Series 6, 41:243, 357-377, DOI: $10.1080 / 14786442108636228$

To link to this article: http://dx.doi.org/10.1080/14786442108636228

曲 Published online: 08 Apr 2009.

Submit your article to this journal $\square$

Џ Article views: 2

Q View related articles $\square$

Citing articles: 3 View citing articles 5 
Active Leposits of Radium, Thorium, and Actinium. 357

so small that it may possibly be due to some extraneous effect due to heating etc.

From these experiments we may therefore safely conclude that neither the black pigment nor the retina as a whole is photo-electric to visual light. It is possible however that, as previously mentioned, the nul effects obtained with the retina may be due to absorption of the electrons in the surface. It remains quite conceivable that the rhodopsin in the eye is actually photo-electric, but so immersed in inactive material that the electrons cannot escape. Thns the photo-electric theory of vision, while still presenting many attractions, cannot be said to be in any way confirmed by these experiments, but neither can it be actually refuted by them. To obtain really conclusive evidence it would be necessary to isolate sufficient rhodopsin in a pure state to be able to test it directly.

In conclusion I wish to express my thanks to Professor Pringle and Dr. Fearon for their kind aid in the preparation of the frogs' retinas; also to Dr. Joly, to whose suggestion and assistance the research is mainly due.

Iveagh' Geological Laboratory,

November 1920.

XXXII. The Nistribution of the Active Deposits of Radium, Thorium, and Actinium in Electric Fields. By G. H. BrIgGs, B.Sc., Lecturer in Physics at the University of Sydney*.

\section{Introduction.}

1) UE experiments to be described in this paper were 1 begun with the object of deciding whether, as E. M. Wellish $\nmid$ had concluded, there is a definite limiting fraction of the recoil atoms from radium emanation, positively charged at the end of the recoil path, or whether, as G. H. Henderson concluded, all the recoil atoms from thorium and radium emanations are positively charged at the end of the recoil path. As the work progressed, it was found necessary to extend its scope.

In his experiments Wellish used a cylindrical vessel with a central electrode. He obtained the following results for the recoil atoms of radium emanation : 88.2 per cent.

* Communicated by the Author.

$\dagger$ Wellish, Phil. Nag. xxviii. p. 417 (1914).

Phil. Mag. S. 6. Vol 41. No. 243. March 1921. 


\section{Mr. Briggs on Distribution of Active Deposits of}

initially positively charged in air and hydrogen, 78.9 in carbon dioxide, the remainder being neutral, and in ether vapour all the deposit atoms were nentral. The term "initial" refers to the instant when the recoil atom bas reached the end of its recoil path, and the values quoted represent the fraction of the recoil atoms which possess a positive charge at the end of this path before either volume or columnar recombination has had a chance to become operative. The chief advantage of the type of vessel used by Wellish is that a delicate test of the presence of negative deposit atoms can be made if the central electrode is positive. In his first paper Henderson * described the results of experiments on thorium active deposit. The electrodes were parallel plates with a guard ring, and were $3 \mathrm{~cm}$. apart. In the air the cathode activities at 120 and 12,000 volts were $98 \cdot 6$ and $99 \cdot 8$ per cent. As the latter value differed from 100 by less than the experimental error, Henderson concluded that all the deposit atoms from thorium emanation are initially positively charged in air. When ether vapour was added to the air the percentage decreased, becoming zero for pure ether vapour. In two succeeding papers, however, Henderson $\dagger$ described experiments on radium emanation, and concluded from the results he obtained when using a new form of parallel plate exposure vessel and strong uniform electric fields, that all the deposit particles from radium emanation in air, carlon dioxide, and sulphur dioxide are positively charged. In column D of Table $I$. are given values calculated from those of Henderson, who expressed his results as the ratio of the cathode activity to the total activity.

As the values were still increasing at the highest potential. used, he concluded that there is no limit to the percentag: cathode activity, and hence that initially all the deposit atoms are positively charged.

\section{Experiments with Radium Emanation in Strong Fields.}

Fig. 1 shows an apparatus designed by the author for use with strong fielde. $A$ and $B$ are two circular brass disks cemented to an ebonite ring $P$. The inner surface of the ring was curved, as shown, to prevent sparking across this surface, and it projected beyond the brass disks to prevent brush discharge and sparking from their edges.

* Henderson, Trans. Nova Scotian Inst. Sc. xiv. p. 1 (1914-15).

$\dagger$ Henderson, Trans. Nova Seotian Inst. Sc. xiv. p. 123 (1916); and Trans. Roy. Soc. Canada, x. p. 151 (1916). 
Radium, Thorium, and Actinium in Electric Fields. $\quad 359$

The central portions, $\mathrm{C}$ and $\mathrm{D}$, of the disks were removable, and could be made air-tight by running soft wax into channels formed by brass rings on either side of the junction-line. The diameter of the portion of the disks

Fig. 1.

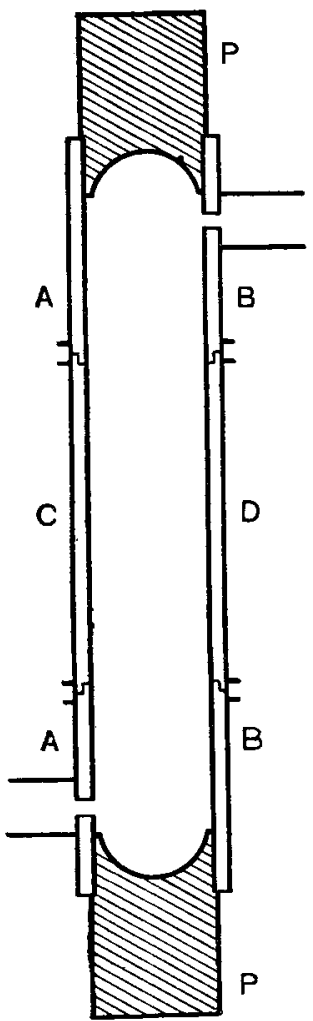

Fi⿳亠丷. 2.

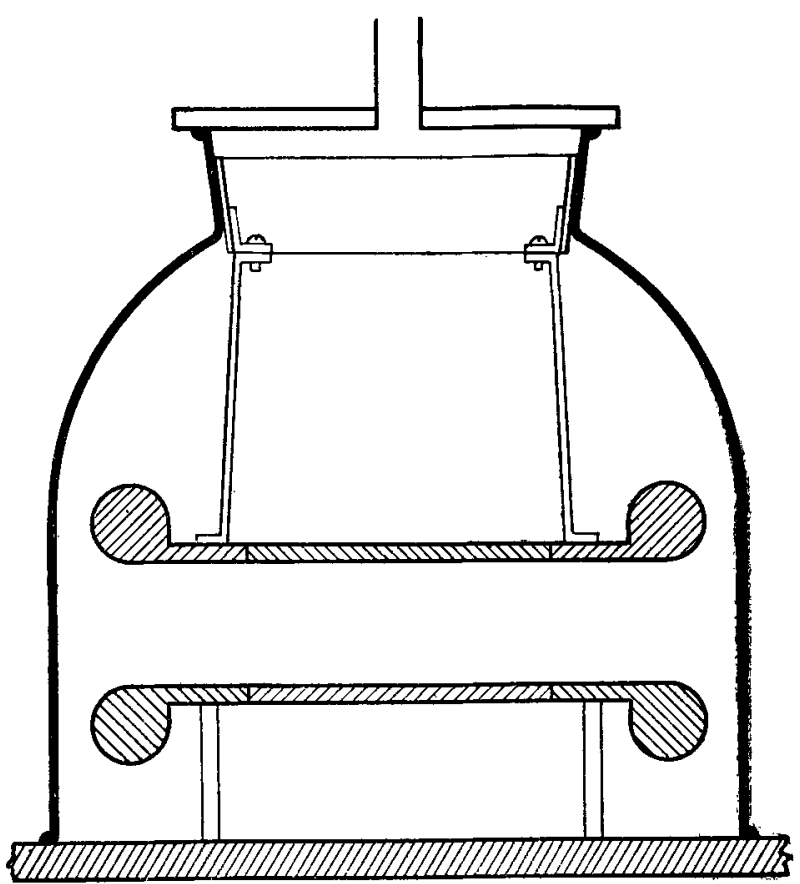

exposed on the inside of the vessel was $10.6 \mathrm{~cm}$., and that of the removable disks $5.6 \mathrm{~cm}$. The plates were $2 \mathrm{~cm}$. apart, and their internal surfaces were cleaned with fine emery paper. Radium emanation was introduced mixed with the gas under observation, or was allowed to accumulate over-night from a small quantity of radium placed inside the vessel. A Wimshurst machine was used for high potentials. The gases were dried and filtered. At the end of an exposure, which was always longer than three hours, the alpha-ray activities of the central disks were compared by means of a Dolezalek electrometer. Wellish *

* Loc. cit.

2 B 2 
3ti0 Mr. Briggs on Distritution of Active Deposits of

showed that the anode activity was due to the diffusion of neutral deposit atoms : hence if $c$ and $d$ are the cathode and anode activities respectively, $\frac{c-d}{c+d}$ would give the fraction of the $\mathrm{RaA}$ atoms positively charged, if, as assumed by Wellish, the recoil of llaB from the electrodes is negligible. This assumption will be shown later to be incorrect. In column A of Table I. are given the values of $\frac{c-d}{c+d}$ calculated from the anode and cathode activities for radium emanation mixed with air. No evidence is shown of a continual increase with voltage, the limiting value being practically reached at 60 volts per $\mathrm{cm}$. The mean value $89 \cdot 6$ is in good agreement with that found by Wellish when using a different type of testing vessel.

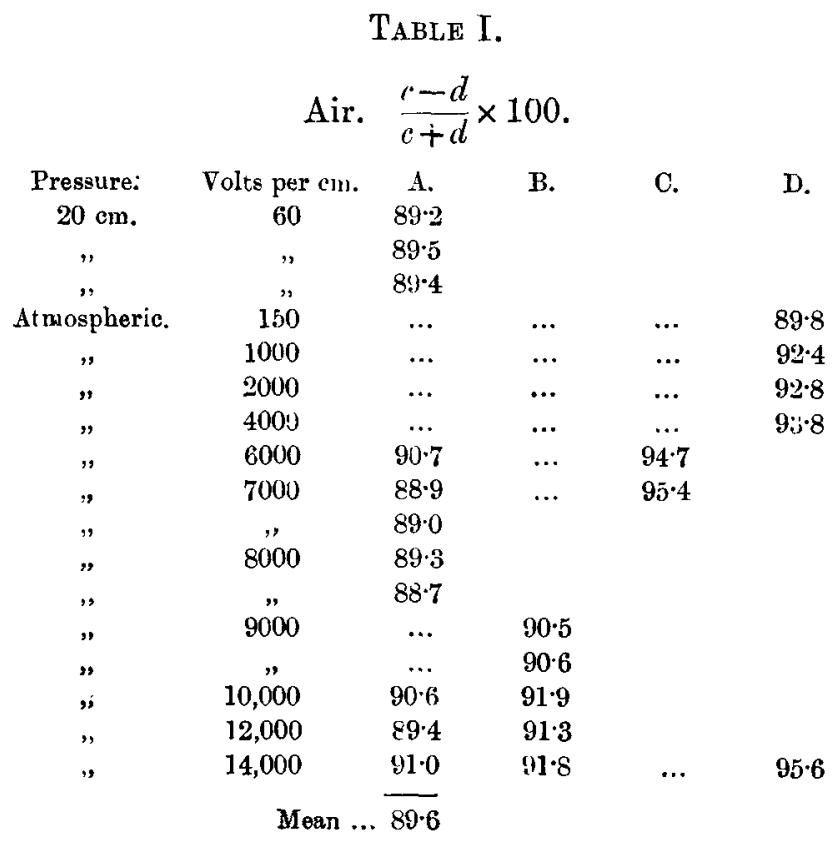

To determine the reason for the discrepancy in the results obtained by the author and by Henderson, experiments were made with an apparatus similar as far as possible tn that used by Henderson. The two results shown in column $C$ sufficed to show that his experimental results 
Radium, Thorium, and Actinium in Flectric Fields. 361

could be reproduced. However, when this apparatus was slightly modified, the increase of the experimental values with the voltage practically disappeared. Fig. 2 shows the modified form of Henderson's apparatus used by the writer. The electrodes were two brass plates $10.4 \mathrm{~cm}$. in diameter, held, as indicated, at a distance of $2 \mathrm{~cm}$. apart in a bell-jar of $11.5 \mathrm{~cm}$. internal diameter. The central portions, whose diameter was $5 \cdot 1$, were removable, and the activities on these were measured. Henderson used flat brass plates separated by three vertical glass rods, the rods and the edges of the plates being covered with paraffin wax to stop brush discharge. $\mathrm{He}$ used cotton wadding to prevent diffusion of active deposit from above or below the plates into the region between them. The following modifications were made by the writer to eliminate to a múch higher degree than was done by Henderson brush discharge near the edges of the plates:-(1) The edges were rounded off as shown in the figure, (2) a roll of silk was used instead of cotton-wool, and (3) the upper plate was supported from above. The results obtained with this apparatus are given in column B. The continual increase lound by Henderson at high voltage has practically disappeared. The difference between the values of column $B$ and the mean of column $A$ is to be ascribed possibly to some brush discharge still being present, and in the case of the last three readings to the fact that for these readings the plates were very highly polished. The importance of keeping the state of polish of the surfaces always the same had not been recognized at this stage of the experiments.

'The high values given by the apparatus used by Henderson were due to the action of brush discharge from the edges of the plates or from cotton-wool fibres. If cotton-wool is held against a pole of a Wimshurst machine in the dark, it glows quite brightly, whereas good silk cloth does not. From any point near the edges of the disks where a brush discharge occurs, the gas is blown on to the opposite electrode, or more probably on to the wall of the bell-jar, to which any neutral deposit atoms carried by the gas will adhere. In a short time all the gas between the electrodes will be entrained in these so-formed jets, and only a fraction of the neutral deposit will settle on the central portions of the plates. As the voltage is increased so will the intensity of the brush discharge, and with it the apparent percentage cathode activity. This action of the brush discharge was illustrated by filling a vessel similar to Henderson's with smoke. At places on the glass wall opposite points on the electrodes 


\section{Mr. Briggs on Distritution of Active Deposits of}

where brush discharge occurred a deposit of smoke was observed.

Further confirmation of Wellish's conclusion that there is a definite limiting value to the percentage of radium active deposit positively charged was wiven by the results found for ethylene. At 720 volts and $20 \mathrm{~cm}$. pressure, using the vessel shown in fig. 1 , the value for ethylene was 23.4 . Higher values were not obtained at voltages as high as 12,000 , using the vessel shown in fig. 2 .

\section{The Diffusion of the Neutral Deposit Atoms.}

The experiments were then continued, using the ebonite ring type of vessel, in an endeavour to discover how the percentage depended on the nature of the gas. The positively cluarged atoms which reach the central disks EF or.$G H$ (fig. 3) are drawn from gas in the cylindrical volume EFHG. A portion, however, of the neutral atoms produced in this volume will diffuse out of it towards the walls $\mathrm{AC}$ and BD. Unless the number that so diffuse is negligible, this type of vessel will yield values for the percentage

Fig. 3.

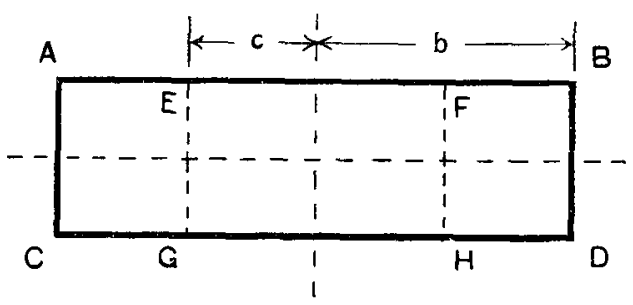

positively charged that are too high. Wellish * showed that the number of neutral deposit atoms at any point in a cylinder is given by the following equation:

$$
n=\frac{q}{4 \mathrm{D}}\left(b^{2}-r^{2}\right)-\frac{2 q}{b \mathrm{D}} \sum_{n=1}^{\infty} \frac{1}{\lambda_{n}^{3}{ }^{3}} \frac{e^{\lambda_{n}{ }^{2}}+e^{-\lambda_{n} z} \mathrm{~J}_{0}\left(\lambda_{n} r\right)}{e^{\lambda_{n} l}+e^{\lambda_{n} l} \mathrm{~J}_{1}\left(\lambda_{n} b\right)},
$$

where $r$ and $z$ are cylindrical co-ordinates, the origin being at the centre of the cylinder and $z$ being measured along its axis.

$b$ is the radius of the cylinder, and $2 l$ its length. $q$ is the number of neutral atoms produced per c.c. by the decay of the emanation, and $\mathrm{D}$ their diffusion coefficient.

* Loc. cit. 
Radium, Thorium, and Actinium in Electric Fields.

$\lambda_{1}, \lambda_{2}, \ldots \lambda_{n}$ are the real positive roots of the equation

$$
\mathrm{J}_{0}(\lambda b)=0 \text {. }
$$

Let $c$ be the radius of each of the central disks EF and GH.

The number of neutral atoms reaching EF or GH per second is

$$
-2 \pi \mathrm{D}\left(\frac{d n}{d z}\right)_{l} \int_{r=0}^{r=c} r d r=\frac{4 \pi q c}{b} \sum_{n=1}^{\infty} \frac{1}{\lambda_{n}^{3}} \frac{J_{1}\left(\lambda_{n} c\right)}{J_{1}\left(\lambda_{n} b\right)} \tanh \left(\lambda_{n} l\right) .
$$

The total number of neutral atoms generated in the cylinder $\mathrm{EFHG}$ is $2 \pi c^{2} l q$, and the ratio of the number reaching the central plates to the number generated betwoen them is

$$
\frac{4}{b c i} \sum_{n=1}^{\infty} \frac{1}{\lambda_{n}^{3}} \frac{J_{1}\left(\lambda_{n} c\right)}{J_{1}\left(\lambda_{n} b\right)} \tanh \left(\lambda_{n} l\right),
$$

an expression which depends only on the dimensions of the containing vessel.

This series converges rather slowly. From the first twelve terms the result found for the vessel with the ebonite ring was 0.993 . A second determination, made by giving $c$ a very slightly different value, was $0 \cdot 989$. Hence all but about one per cent. of the neutral atoms formed in the volume EFHG reach the plates $\mathrm{EF}$ and GH. 'The error introduced is therefore negligible, and has been neglected in all subsequent calculations.

In the case of the apparatus described by Henderson, for which $2 b=11.5 \mathrm{~cm} ., 2 c=7 \cdot 0 \mathrm{~cm}$., and $2 l=3 \mathrm{~cm}$., values calculated were $0 \cdot 965$ and $0 \cdot 952$. Values of $J_{1}$ were obtained from a table given by Wilson and Peirce**

\section{Experiments with Radium $B$.}

Previous investigators on the distribution of radium and thorium active deposits in electric fields have neglected the part played by the disintegration of $\mathrm{RaA}$ into $\mathrm{RaB}$ and $\mathrm{ThA}$ into ThB. It will be shown subsequently that if the percentage of $\mathrm{RaB}$ which is initially positively charged at the end of the recoil path in a particular gas, and also the efficiency of recoil of $R a B$ from $R a A$ from the surfaces of the electrodes are known, then the percentage of RaA initially positively charged in that gas may be calculated from the results of experiments in which the gas is mixed with radium emanation.

* Wilson and Peirce, Bull. Amer. Math. Soc. iji. p. 153 (1896-7). 
364 Mr. Briggs on Distribution of Active Deposits of

Also, by making use of the fact that $\mathrm{RaA}$, ThA, and AcA are isotopes, as also are $\mathrm{RaB}$, ThB, and $\mathrm{AcB}$, the difference in the distribution of radium, thorium, and actinium emanations in an electric field may be quantitatively, explained. The experiments by which the percentage of $\mathrm{RaB}$ initially positively charged in various gases, and the efficiency of recoil of $\mathrm{RaB}$ from the electrodes were measured, will now be described.

The experiments consisted in collecting $\mathrm{RaB}$ by recoil from a plate made active with RaA, first in the gas under observation and then in air. This gave the ratio of the fraction of $\mathrm{RaB}$ initially positively charged at the end of the recoil path in the gas, to the corresponding fraction for air. In a second experiment the fraction for air was found.

Two metal plates $6.7 \mathrm{~cm}$. in diameter were separated by a vertical ebonite plate $1.5 \mathrm{~cm}$. thick, having a hole $4.8 \mathrm{~cm}$. in diameter in the centre. Stop-cock grease was used to make the contact surfaces air-tight. Through a three-way tap, air or the gas under observation having passed through drying and filtering tubes could be admitted. Another three-way tap led to a pump and manometer. When the active plate was negatively charged, or if there was no field between the plates, and the vessel filled with a dry dust-free gas, the opposite plate received a negligible amount of $\mathrm{RaB}$, which was probably chiefly due to the initial disturbance caused by exhausting and filling the vessel, and not to the presence of negative RaB atoms. All the gases used in these experiments were tested for negative atoms by means of exposures in cylindrical vessels, in which the gas was mixed with radium emanation, and in all eases the activity on the central rod when positive was no greater than the amount that should have reached it by the diffusion of neutral atoms. There is no evidence, therefore, of the presence of either negative RaA or RaB atoms. In the experiment with the plates uncharged a layer of neutral $\mathrm{RaB}$ atoms is formed close to the active plate, and the result obtained shows that practically all the neutral atoms return to this plate. A large fraction, depending on the gas in the vessel, of the total activity, was deposited on the cathode when the active plate was positive. It was found that this amount with the pressure of the gas at $15 \mathrm{~cm}$. did not increase perceptibly when the voltage was increased from 160 to 720 .

In an experiment three plates were used. Plate (1) was activated with $\mathrm{RaA}$ by exposure to radium emanation for about half a minute, the portion activated being an area 
$2 \mathrm{~cm}$. in diameter in the centre of the plate. Plate (2) h:tving been placed on one side of the ebonite plate and charged positively, (1) was placed in position opposite it and charged negatively. The air was pumped out and the gas under observation was admitted and pumped out tive times, and the pressure finally adjusted to about $15 \mathrm{~cm}$. The field was then reversed and a stop-watch started. The ictivation of plate (2) was continued for five minutes, at the end of which time the field was again reversed so that (2) ceased to receive $R a B$ from (1). Plate (2) was quickly removed and plate (3) substituted in its place, and the gas pumped ont and replaced by dry filtered air at about $15 \mathrm{~cm}$. pressure. This change could be made within one minute, and when the stop-watch registered six minutes the field was reversed and plate (3) activated for five minutes. When exbausting the vessel, the pressure was not allowed to fall below $1 \mathrm{~cm}$., in order that $\mathrm{RaB}$ should not recoil directly on to (2) or (3). The maximum alpha-ray activities of plates (2) and (3) were compared. Since the amount of RaA on (1) falls to one-quarter in six minutes,

$$
\begin{aligned}
& \frac{\text { Percentage of RaB positively charged in the gas }}{\text { Percentage of } \mathrm{RaB} \text { positively charged in air }} \\
& =\frac{\text { maximum activity of }(2)}{4 \times \text { maximum activity of }(3)} .
\end{aligned}
$$

The results obtained for various gases are given in column 2 of Table II. No alteration in the results was found when air was admitted in the first half of the experiment and the gas in the second half, or when the activity of plate (1), and hence the ionization between the plates, was increased by long exposure to the emanation to ten times its usual amount. In these experiments brass plates were used, except for ammonia, acetylene, and hydrogen-sulphide, for which the plates were of mild steel. Their surfaces were cleaned with fine emery paper.

In experiments on the distribution of the active deposits of radium and thorium emanations the transference from the anode to the cathode of positively charged $\mathrm{RaB}$ or ThB atoms, which were originally neutral $\dot{R}_{a} A$ or ThA atoms, must be considered. It was accordingly necessary to measure the efficiency of recoil from surfaces similar to those used in the experiments with radium emanation. These surfaces had been cleaned with fine emery paper, except in the case of the bell-jar apparatus, in which, as noted previously, they were highly polished. 


\section{Mr. Briggs on Distribution of Active Deposits of}

A clean plate having been placed on the ebonite plate and charged negatively, a second plate was exposed for ten seconds to radium emanation and quickly placed opposite the first, the action of a spring immediately connecting it to the positive pole of the battery (720 volts). Dry filtered air was drawn through the apparatus for about one minute, the final pressure being about $15 \mathrm{~cm}$. The time that elapsed between removing the active plate from the emanation and putting it in position was usually about five seconds. In this case 0.033 of the RaA has decayed before the active plate is in position. At the end of 27 minutes the two plates were removed, and their activities compared by means of a Dolezalek electrometer of sensitivity $2000 \mathrm{~mm}$. per volt. The results obtained, after correcting for the initial decay of the RaA, for the efficiency of recoil in air of $\mathrm{RaB}$ from plates of brass, steel, and zinc, cleaned with emery paper, were respectively $81 \cdot 7,73$, and 73.9 per cent. The values given by different readings remained constant to within aliout 2 per cent.

\section{The Percentage of RaB positively charged in Air.}

The percentage of RaB initially positively charged in air was measured by modifying the method described above for comparing gases with air. It was arranged that the RaB atoms leaving plate (1) during the first 5 minutes' exposure should be received by a disk fastened to plate (2), so that it was separated from plate (1) by a distance of $0.06 \mathrm{~mm}$. Mica on the edge of the disk prevented contact with (1). A potential of 160 volts was maintained between plate (1) and the disk, the latter being negative. Plate (2) and the disk were placed in position first, and the pump started. Plate (1), with its deposit of RaA, was then placed in position, and the stop-watch started. The pump was kept running throughout the exposure and rapidly produced a vacuum of $0.01 \mathrm{~mm}$. At the end of the five minutes air was admitted and the disk quickly removed. An exposure was then made with plate (3) opposite plate (1) from the 6 th to the 11th minute in air at $15 \mathrm{c:n}$. pressure at the original distance of $1.5 \mathrm{~cm} .$, using 720 volts. Since the range of recoil of $\mathrm{RaB}$ at $0 \cdot 01 \mathrm{~mm}$. pressure is several metres, all the RaB atoms shot off from the plate should be collected during the first exposure. The mean result found by this method was $93 \pm 0^{\circ} 6$ for the percentage of $\mathrm{RaB}$ recoil atoms initially charged in air. By multiplying: the values given in column 2 by 0.93 , the corresponding 
Radium, Thorium, and Actinium in Electric Fields. 367

values for the other gases are obtained. These are given in column 3. When the values given above for the efficiency of recoil in air are divided by 0.93 , the efficiency of recoil is given in a form which is independent of the gas in to which the atoms are projected and which depends only on the nature and surface of the metal, the values so obtained are: for the brass plates $87 \cdot 8$, for the steel 78.5 , and for zinc $79 \cdot 5$.

It has been assumed in the foregoing that the total amount of $\mathrm{RaB}$ recoiling from a plate is independent of the gas in contact with it. It is difficult also to estimate the reliability of the result 93 per cent. for $\mathrm{RaB}$ in air, as it is unknown whether reflexion of $\mathrm{RaB}$ from the cathode or trom residual gas occurs to any large extent during the first exposure. However, it appears from the results found later that the percentage for $R: B$ in any gas is always much greater than that for $R \mathrm{~A} A$, and therefore that the value in air for $\mathrm{RaB}$ is much greater than 82 .

\section{The Distribution of the Active Deposits of Radium, Thorium, and Actinium Radiations in Electric Fields.}

The distribution of the activity on the two electrodes is the result of several distinct processes.

1. The disintegration of the emanation gives rise to A-atoms, definite fractions of which are positive and neutral at the end of the recoil path.

2. The positive A-atoms are drawn by the field towards the cathode, and a fraction of them transform on the way into positive or neutral $\mathrm{B}$-atoms. Of the former, owing to the comparatively long life of the B-atoms of the three radioactive series, practically all reach the cathode as B-atoms. The neutral B-atoms reach the electrodes by diffusion.

3. The neutral A-atoms produced by process (1) diffuse in equal numbers towards each electrode, and a fraction of them transform on the way into neutral or positive B-atoms, the former going to the cathode and the latter diffusing in equal numbers to each electrode.

The A atoms which have reached the electrodes by process (2) or (3) transform into B-atoms, and a portion of these, depending on the efficiency of recoil from the surfaces, are projected into the gas, a fraction being positive and the remainder neutral at the end of the recoil path. The former are drawn to the cathode, and since the recoil path is a small fraction of a millimetre in these experiments, 


\section{Mr. Briggs on Distribution of Active Deposits of}

it is held that all the nentral B-atoms return by diffusion to. the surface from which they are projected.

Direct recoil on to the electrodes is negligible in the experiments of Wellish, Henderson, and the author. The magnitude of the effect produced by processes ( 2 ) and (3) depends on the transformation constants of the A-atoms of the three radioactive series of elements. Notransformations after that of $\mathrm{A}$-into $\mathrm{B}$-atoms need be considered, since $\beta$-recoil of (-atoms is entirely negligible in these experiments, and the products of the $\mathrm{C}$-atoms contribute a negligible amount to the ionization by which the activity of the electrodes is measured.

In a theory which will be given later, it is held that the last few collisions at the end of the recoil path determine whether the recoil atom will be positive or neutral. If this is so, the percentage initially positively charged in a gas will be the same for $R a A$, ThA, and AcA, since they are isotopes, although their initial velocities are different. In the same way, the percentages for $\mathrm{RaB}, \mathrm{ThB}$, and $\mathrm{AcB}$ will be identical and independent of whether the transformation takes place in the body of the gas or if the atom recoils into the gas from an electrode.

The fraction of radium and thorium positive A-atoms which transform into $B$-atoms before reaching the cathode is negligible in the experiments described here. The fraction of the neutral $A$-atoms which so transform in a parallel plate vessel may be found as follows :-In section III. it was proved that the diffusion of neutral atoms between the central disks is very approximately linear along the $z$-axis.

Let $\mathrm{D}$ be the coefficient of diffusion of the $\mathrm{A}$ atoms, and $\lambda$ their transformation constant. $q$ and $z$ have the same meanings as before.

Let $n$ be the number of neutral A-atoms per c.c. at a distance $z$ along the $z$-axis.

We have

$$
\mathrm{D} \frac{d^{2} n}{d z^{2}}=\lambda n-q .
$$

Writing $y=\lambda n-q$, the equation becomes

$$
\mathrm{D} \frac{d^{2} y}{d z^{2}}=\lambda y
$$

the solution of which is

$$
\cosh \left\{\frac{\lambda^{\frac{1}{2}}}{\mathrm{D}^{\frac{1}{2}}}\left(z+a_{1}\right)\right\}=a_{2} y
$$

where $a_{1}$ and $\alpha_{2}$ are constants. 
Radium, Thorium, and Actinium in Electric Fields. 369

It is readily found from the boundary condition, viz. $\approx=0$ when $r= \pm l$, that

$$
a_{1}=0 \text { and } a_{2}=\frac{-\cosh \left(\frac{\left.\lambda^{\frac{1}{2}} l\right)}{\bar{D}^{\frac{2}{2}} l}\right)}{q} .
$$

Let $s$ be the fraction of the neutral A-atoms produced in the gas that reaches the central electrodes before trinsforming into B-atoms. Then

$$
\begin{aligned}
s & \left.=-\frac{\mathrm{D}\left(\frac{d n}{d z}\right)}{q^{l}}\right) \\
& =\frac{\mathrm{D}^{\frac{1}{2}}}{l \lambda^{\frac{1}{2}}} \tanh \left(\frac{l \lambda^{\frac{1}{2}}}{\bar{D}^{\frac{1}{2}}}\right) .
\end{aligned}
$$

Wellish * found 0.045 for the coefficient of diffusion of the neutral deposit atoms of radium emanation in air at itmospheric pressure. $\mathrm{H}_{\theta}$ neglected, however, the effect of the recoil of $\mathrm{RaB}$ during his experiments. On taking this into account, the writer has found that Wellish's results lead to the value 0.056 . Using this value, for $\mathrm{RaA}$ in air at atmospheric and at $20 \mathrm{~cm}$. pressure, in the vessel shown in fig. $1, s=0.978$ and 0.994 respectively. The corresponding values for ThA are 0.107 and 0.207 , and for AcA 0.018 and 0.025 . At the latter pressure the amount of RaA transforming before reaching the electrodes may be neglected.

\section{Radium $A$.}

The percentage of $\mathrm{RaA}$ initially positively charged in a gas may be deduced from the value of $\frac{c-d}{c+d}$ found in an experiment in which the gas is mixed with radium emanation, and from the percentage of RaB initially positively charged at the end of a recoil path in that gas.

Let $a=$ the fraction of $\operatorname{RaA}$ atoms initially positively charged.

$b=$ the fraction of $\mathrm{RaB}$ atoms initially positively charged. (Column 3, Table II.)

$r=$ the efficiency of recoil of $\mathrm{IaB}$ from the electrodes. This number includes both the positive and neutral atoms.

$p_{1}=\frac{c-d}{c+d}, c$ and $d$ being the cathode and anode activities in an exposure in which the gas is mixed with radium emanation.

* Loc. cit. 
$370 \mathrm{Mr}$. Briggs on Ihistribution of Active Deposits of

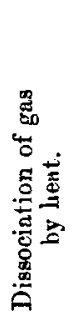

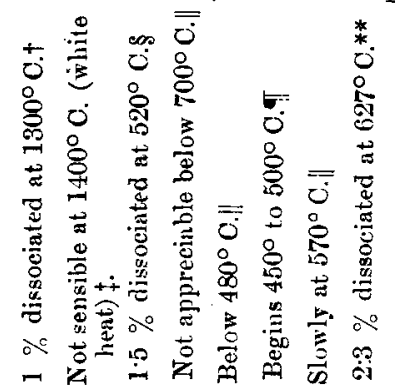
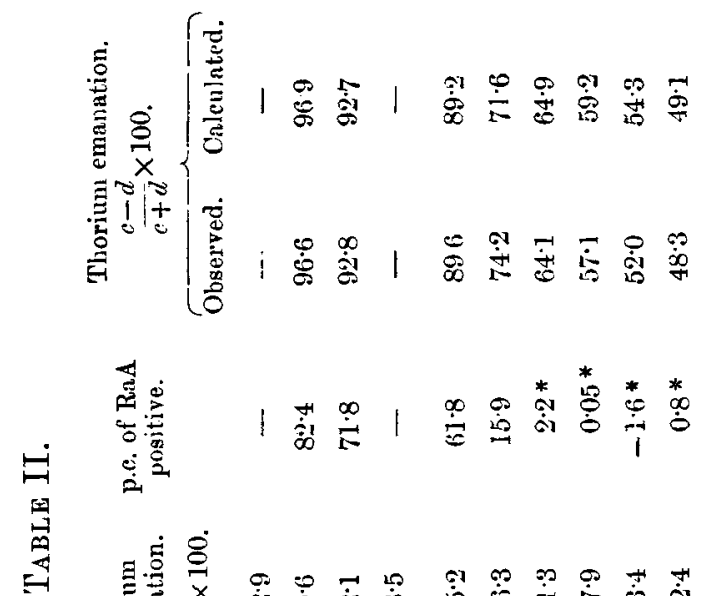

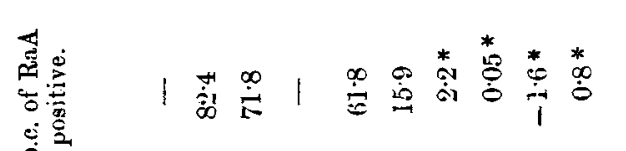
$\dot{1}$

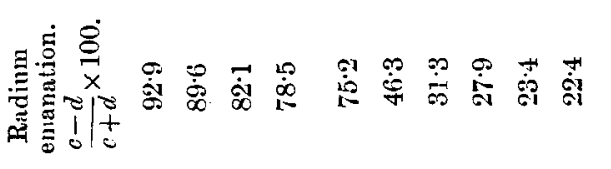

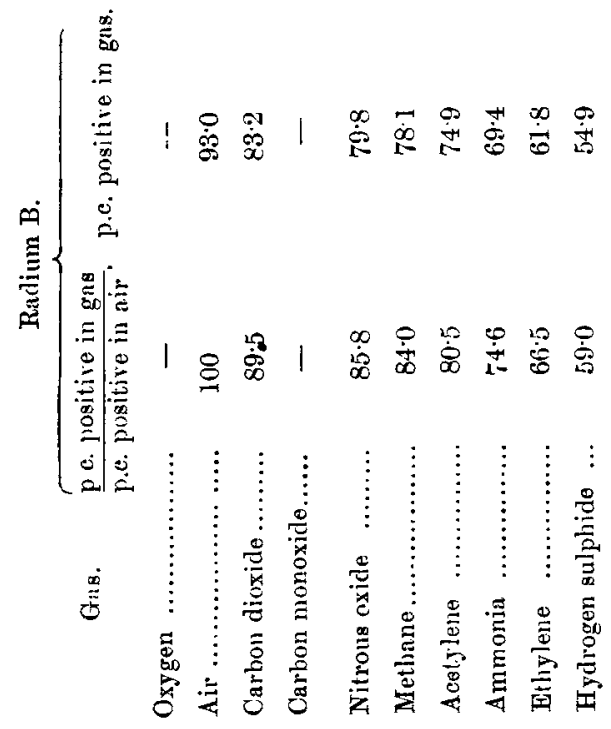


Radium, Thorium, and Actinium in Electric Fields. 371

Let us consider the activity accumulating on the anode. It receives by diffusion half of the neutral $\mathrm{A}$ atoms, i.e. $\frac{1-a}{2}$. Of these, $\frac{r}{4}(1-a)$ free themselves by recoil, and $\frac{b r}{4}(1-a)$ of them are initially positively charged and are drawn to the cathode. The neutral atoms return to the anode by diffusion. Hence the total number of atoms left on the anode is $\frac{1}{2}\left(1-\frac{b r}{2}\right)(1-a)$. The rest of the atoms resulting from the decay of the emanation reach the cathode. It is readily found that

$$
a=\frac{2 p_{1}-b r}{2-b r} .
$$

Since $b$ is obtained by multiplying the experimental values given in column 3 by 0.93 , and $r$ is obtained by dividing the value found for the efficiency of recoil found in air by the same number, then $b r$ and hence the above equation are independent of the experiment in which 93 per cent. was obtained for $\mathrm{RaB}$ in air. The values of a calculated from this equation are given in column 5 .

\section{Thorium Emanation.}

To test the theory given for the distribution of the active deposits, exposures were made with thorium emanation mixed with various gases, using the vessel shown in fig. 1 . The relative difference in mass of the isotopes of $\mathrm{RaA}$ or of RaB is so small that we may assume that for each group of these isotopic atoms the percentage positively charged, the coefficient of diffusion, and the efficiency of recoil are identical. Hence it is easily shown that $p_{2}$, the value of $\frac{c-d}{c+d}$ for thorium emanation, is given by

$$
p_{2}=a+b(1-a)\left(1-s+\frac{s r}{2}\right) \text {. }
$$

It is to be noted that $p_{2}$, owing to the terms involving $s$, depends on the size and the type of testing vessel used and also on the pressure of the gas. The values of $p_{2}$ found by experiment and by means of this equation are given in columns 6 and 7 . It was assumed that the coefficient of diffusion of the A-atoms is inversely proportional to the square. 


\section{$372 \mathrm{Mr}$. Briggs on Nistribution of Active Deposits of}

root of the molecular weight of the gas through which diffusion takes place. The experiments with radium and thorium emanations were made at a pressure of $20 \mathrm{~cm}$., the time of exposure being generally greater than 16 hours, and at a voltage of 720 , except in the case of hydrogen sulphide, for which consistent results for either emanation or in the rxperiments with $\mathrm{RaB}$ were only obtained when voltages as high as 10,000 were used. Zinc electrodes were used for the gases ammonia, acetylene, and hydrogen sulpbide. Each result given in Table II. is the mean of several experiments. It was found necessary to liquify and fractionate the gases methane and ethylene to free them from the vapours of organic compounds produced during their preparstion. Hydrogen sulphide was obtained by heating a solution of magnesium hydrosulphide, and was also fractionated.

\section{Actinium Active Deposit.}

The experiments of Kennedy * Lucian + , or of McKeehan $\ddagger$ might be used to test the theory given above for the case of actinium emanation. Lucian's experiments are by far the most suitable for this purpose, since direct recoil on to the electrodes was negligibie in his but not in the experiments of the other two. Lucian's method was very similar to that used by Wellish for radium active deposit. In section V. it has been shown that the number of neutral atoms of AcA diffusing to the walls of a vessel with parallel plates $2 \mathrm{~cm}$. apart, before transforming into $A c B$, is 1.8 per cent. at atmospheric pressure. The number so diffusing, in the ressel used by Lucian, which was a cylinder of $4.9 \mathrm{~cm}$. diameter with a central rod as cathode, will be of the same order of magnitude and may be neglected. By means of a formula given by Lucian, the amount of AcA positively charged which reaches the cathode before transforming into AcB may be calculated. Taking 1.2 for the mobility of AcA, the fraction of the AcA positively charged reaching the cathode in Lucian's vessel, before transforming into $\mathrm{AcB}$, is 14 per cent. at 600 volts, 17 at 980 volts, and 20 at 1700 volts. Let us consider 100 molecules of actinium emanation. The first transformation produces $100-82 \cdot 4$ or $17 \cdot 6$ initially neutral atoms of AcA. Practically all of these break up before diffusing to the walls, and 100-93 or 7 per cent. of them, i. e. $1 \cdot 23$, become neutral atoms

* Kennedy, Phil. Mag. xviii. p. 744 (1909).

+ Lucian, Phil. Mag. xxviii. p. 761 (1914).

$\ddagger$ MeKeehan, Phys. Rev. x. p. 473 (1917). 
Radium, Thorium, and Actinium in Electric Fields. 373

of AcB. Of the original $82 \cdot 4$ positive A-atoms, 86 per cent. at 600 volts break up before reaching the cathode, and therefore $82.4 \times 0.86 \times 0.07$ or 4.96 become neutral $\mathrm{B}$-atoms. The total number of nautral $\mathrm{B}$-atoms is then $6 \cdot 19$, and since the half-transformation period of $\mathrm{AcB}$ is 36 minutes, they all diffuse to the walls and remain there at the $\beta$-transformation to AcC. Lucian found, for the smallest quantity of emanation used, $6 \cdot 3$ per cent. neutral, volume recombination being apparently not entirely absent. At 980 he found values ranging from $5 \cdot 3$ to $5 \cdot 0$, while the value calculated as above is 5.9 ; at 1700 he found $5 \cdot 1$ per cent. neutral, while the culculated value is $5 \cdot 8$. It is to be noted that the neutral B-atoms resulting from the disintegration of positive A-atoms have been carried some distance towards the cathode, and hence the cathode receives a greater percentage of the neutral atoms while the field is on than when there is no field. The values found by Lucian are calculated on the assumption that the cathode receives the same percentage of neutral atoms in both cases, and hence they are too small. Lucian's results are therefore consistent with the general explanation a dvanced for the distribution of the active deposits.

\section{Discussion of the Results.}

These experiments confirm Wellish's conclusion that there is a definite limiting value to the percentage of the active deposit initially positively charged in a gas, and that the anode activity is due to the diffusion of neutral uncharged deposit. The latter conclusion is at variance with that of Ratner*, who maintained that the anode activity is due to the carriage to it by an electric wind of deposit particles which have lost their charge. It is difficult, however, to see how there can be any wind involving the motion of neutral gas molecules or neutral deposit atoms in an enclosed parallel plate vessel such as used in the present experiments. The gas-pressure acting on the two plates is certainly increased as a result of the electric force acting on the positive and negative ions; but there will be no wind unless these forces are unbalanced, as, for example, if the plates were perforated, or as in the case of discharge from a point. While there is no doubt that many of Ratner's experimental results were due to an electric wind, quite a different explanation is suggested for those made in the glass tube with disk electrodes, and which have most bearing on the present subject. It is possible that the large amount of emanation used per c.c., which was

* Ratner, Phil. Mag. xxxiv. p. 429 (1917).

Phil. Mag. S. 6. Vol. 41. No. 243. March 1921. 2 ( 


\section{Mr. Briggs on Distribution of Active Deposits of}

about twenty times that at which Wellish* showed that aggregation set in, together with the additional strong source of alpha-ray ionization, resulted in the production of aggregates of deposit atoms which are known to be able to take positive or negative charges. They are likely then to assume a positive or negative charge according as to whether they are in a region in which there is an excess of positive or of negative ions. The additional source of alpha-ray ionization, together with the separation of the jons by the electric field, would produce a distribution of positive and negative ions in the gas which would account for the results on this theory.

On searching for a general law connecting the resilts obtained and the properties of the various gases, it was seen that the percentage of either $\mathrm{RaA}$ or $\mathrm{RaB}$ positively charged was generally high for those gases whose heat of formation was large. However, a closer relationship appeared to exist between the results and the stability of the gases for increase of temperature: this latter quality, of course, depends partly on the heat of formation. In the table, data estimated from the results of various observers are given concerning the temperatures at which the various gases begin to dissociate. For the gases investigated, the percentage of recoil atoms positively charged decreases with the temperature of dissociation of the gas. It is well known that alpha-rays decompose many gases, and as the initial energy of a recoil atom is of the order $10^{10}$ times the average energy of a gaseous molecule at $0^{\circ} \mathrm{C}$., it is to be expected that a recoil atom will dissociate many of the molecules in its path, even in the case of elementary gases such as hydrogen and oxygen, and that a part at least of the observed dissociation is produced in this way. The dissociation of gases by heat is usually a bimolecular process; whereas if dissociation by recoil atoms occurs, it will probably consist in the disruption of single particles. Hunter $\dagger$, for example, has shown that the dissociation of nitrous oxide by heat is represented by the equation

$$
2 \mathrm{~N}_{2} \mathrm{O}=2 \mathrm{~N}_{2}+\mathrm{O}_{2}
$$

and Wourtzel $\ddagger$ gives reasons for believing that in the case of alpha-rays some of the gas is disenciated according to the equation

$$
\mathrm{N}_{2} \mathrm{O}=\mathrm{NO}+\mathrm{N} \text {. }
$$

If a bi-atomic molecule, for instance one of carbon monoxide,

* Wellish, Phil. Mag. xxviii. p. 417 (1914).

+ Hunter, Zeit. Phys. Chem. liii. p. 441 (1005).

t Wourtzel, Le Radium, xi. p. 333 (1919). 
is dissociated by a recoil atom, a free atom of oxygen and one of carbon will be produced. The heat of formation of bimolecular oxygen from atomic oxygen has been estimated by Lewis * to be about $14 \times 10^{\star}$ calories per gram-molecule. The work done, therefore, in dissociating single molecules will be much greater than when the dissociation is bimolecular. It is considered, therefore, that the dissociation of a gas by heat is only an approximate guide to the stability of the gas under the bombardment of recoil atoms.

A qualitative explanation of the results obtained may be given if the following assumptions are made :-

1. At a collision between a recoil atom and a molecule the former may become positively charged if neutral before the collision, if the relative velocity of the two is greater than a certain value, which depends on the nature of the recoil atom and of the gas.

2. If the relative velocity is greater than this amount, the recoil atom has a $\operatorname{large} \theta$ chance of being positively charged immediately after a collision in which the gas molecule is not dissociated. If positive before such a collision, it generally retains its charge.

3. At a collision which dissuciates the gas molecule, the recoil atom has a large chance of becoming veutral.

According to these assumptions, the last collision causing dissociation may leave the recoil atom neutral, and may reduce its velocity to zero or to an amount which is too small to allow the recoil atom to ionize itself and become positively charged. Hence some neutral atoms are likely to be present in all gases except those which are monatomic. If the minimum velocity at which dissociation can be effected is much greater than that at which the recoil atom can ionize itself, then its chance of being positive at the end of its path is large. If, however, the former velocity is less than the latter, all the atoms would be neutral if we were to assume that dissociation occurs at every collision, provided the velocity is sufficient and that the atom is never positive after such a collision. These two assumptions are not at all likely to be correct; but if the former velocity is very much less than the latter, values approaching zero would be expected from the assumptions previously made. In support of assumption (3), it is suggested that on the disruption of a molecule one or more electrons may be freed which are liable to be captured by a positively charged recoil atom. Also, a head-on dissociating collision will probably be inelastic, the recoil atom penetrating the molecule and

* Lèwis, J. Chem. Soc. Trans. cxv. p. 182 (1919). 
376 Active Deposits of Radium, Thorium, and Actinium.

thus having a large chance of neutralizing itself. Assumption (1), besiles appearing necessary in order to explain the present results, is a natural deduction from Wertenstein's * observation that $\mathrm{RaD}$ recoiling from $\mathrm{RaC}$ in very low vacuo is uncharged at the moment of expulsion, and that the rate of acquisition of a positive charge increases with the pressure.

The ionization potential of the elements increases generally from left to right in the periodic table. From this it is to be expected that the ionization potential of $\mathrm{RaA}$ is greater than that of $\mathrm{RaB}$. If a similar relationship holds for ionization by collision with molecules, then the theory given would require that the percentages for $\mathrm{RaA}$ should be less than for $\mathrm{RaB}$, as is found in all the gases examined, though it is quite possible that the effect is due to a difference in dissociating power of the two.

\section{Summary.}

1. Wellish's conclusion that a definite percentage of the active deposit atoms from radium emanation is initially positively charged at the end of the recoil path, the remainder being neutral, has been verified; and the error in Henderson's experiments, from which he concluded that all the deposit atoms are initially positive, pointed out.

2. The importance of the part played by the recoil of $\mathrm{RaB}$ from the electrodes has been indicated, and the percentages of $\mathrm{RaB}$ initially positively charged in various gases have been measured; and from this the percentages of RaA positively charged calculated from experiments with radium emanation

3. An explanation of the difference in distribution on the anode and cathode of the active deposits of radium, thorium, and actinium has been given on the assumption that the percentages of $\mathrm{ThA}$ and $\mathrm{AcA}$ and of $\mathrm{ThB}$ and AcB initially positive in a gas are identical with those of $\mathrm{RaA}$ and $\mathrm{RaB}$ respectively, as is to be expected from their isotopic nature. The distribution of thorium active deposit was experimentally determived for eight gases in a parallel plate vessel, and found to be consistent with the theory, as also were Lucian's results for the distribution of actinium active deposit in air in a cylindrical vessel.

4. An apparent connexion between the percentage of recoil atoms initially positive in a gas and the temperature

* Wertenstein, C. R. ctxi. p. 696 (1915). 
at which the gas begins appreciably to dissociate has been indicated, and a theory, based on the assumption that the molecules of a gas are decomposed by the deposit atoms during their recoil, has been given to explain the behaviour of the deposit atoms.

I have to thank very sincerely Mr. E. M. Wellish, a suggestion of whose led to this work being undertaken, for his continual interest and advice.

The Physical Laboratory,

The University,

Sydney, N.S.W.,

October 13,1920 .

XXXIII. Magnetic Field of Circular Currents. By H. Nagaoka, Professor of Physics, Imperial University, T'okyo*.

\section{[Plate VI.]}

WANY years ago $I \dagger$ have shown how Э-functions are suited for calculating the strength of the magnetic field due to a circular current. By means of the same functions, the inductances of circular coils can be easily expressed by formulæ developed in $q$-series, which are rapidly convergent. In the present paper the expansion in $q$-series is applied to obtain expressions for the magnetic force of a single coil and of Gaugain-Helmholtz coils, at points not far distant from the axis, in powers of the coordinates.

It is usual to have recourse to expansion in spherical harmonics for expressing the magnetic force of circular currents; but as each harmonic contains different powers of coordinates calculated from the centre of a single coil, or from the middle point of the axis of double coils, the calculation of the deviations of the magnetic force from that at the middle point cannot be easily expressed in power series of the coordinates. By the expansion in $q-$ series, we can arrange terms giving the deviations according to their importance in disturbing the field, and map out the distribution of these perturbations. The study of the curves of equal deviations will be of practical use for finding to what degree of accuracy the field can be assumed as uniform.

* Communicated by the Author.

+ Phil. Mag. vi. p. 19 (1903); Bull. Bureau Stand. xiii. pp. 269-393 1911); Phil. Mag. xxxv. p. 13 (1918). 